\title{
L'inhibition de l'activité PtdIns 3-kinase : un traitement pharmacologique potentiel de la myopathie myotubulaire
}

\author{
Candice Kutchukian, Ana Buj-Bello, Vincent Jacquemond
}

La myopathie myotubulaire est une maladie pédiatrique fatale due à des mutations dans le gène codant la myotubularine (MTM1), une phosphoinositide 3-phosphatase. Elle se caractérise par une faiblesse musculaire très sévère à la naissance ainsi qu'une détresse respiratoire limitant l'espérance de vie à moins d'un an pour la plupart des patients. Le modèle murin déficient en MTM1 (Mtm1-KO) reproduit les principales caractéristiques de la pathologie humaine, dont une faiblesse musculaire conduisant à la mort des animaux autour de 8 semaines. Dans un récent travail, nous avons identifié pour la première fois des altérations de fonction des cellules musculaires de souris Mtm1-KO qui jouent un rôle critique dans la physiopathologie de la maladie. De plus, nous avons révélé le bénéfice d'un traitement pharmacologique. Plus précisément, nous avons démontré que les altérations de signalisation calcique associées à la maladie sont hétérogènes au niveau subcellulaire, et qu'elles affectent non seulement l'amplitude mais aussi les cinétiques d'activation du flux de calcium qui génère la contraction. Nous avons par ailleurs montré que l'inhibition pharmacologique de l'activité phosphoinositide 3-kinase corrige de manière très substantielle les défauts de signalisation calcique in vitro et prolonge l'espérance de vie des souris déficientes en MTM1. La perturbation du métabolisme des phosphoinositides joue donc un rôle critique dans la pathologie et nos résultats fournissent une preuve de concept de bénéfice thérapeutique d'une approche pharmacologique.

Chez l'Homme, différentes mutations dans le gène MTM1 (situé sur le chromosome X) codant la myotubularine, provoquent la myopathie myotubulaire : il s'agit d'une maladie caractérisée par une réduction des mouvements chez le fotus et une faiblesse musculaire très sévère à la naissance. Environ $50 \%$ des enfants atteints ont une espérance de vie inférieure à 1 an, en raison d'une détresse respiratoire [1]. En 2002, l'équipe du Dr Buj-Bello a généré un modèle murin de la myopathie [Mtm1-knockout (-KO)] reproduisant les principales caractéristiques de la maladie humaine : en effet chez la souris, l'absence de MTM1 se caractérise par une faiblesse musculaire évidente dès 3-4 semaines après la naissance et qui progresse ensuite rapidement pour conduire à la mort des mutants, lesquels survivent rarement au-delà de 7 semaines [2].

MTM1 est une phosphoinositide (PtdInsP) 3-phosphatase dont les substrats sont le PtdIns $3 P$ et le PtdIns $(3,5) P_{2}$. Les PtdInsPs sont de petites molécules impliquées dans de nombreux processus cellulaires ; ils se composent d'un cycle inositol et de deux chaînes d'acides gras permettant leur insertion dans les membranes (Figure 1A). La famille des PtdInsPs compte sept espèces inter-convertibles par phosphorylation/déphosphorylation en positions 3, 4 et 5 du cycle inositol. Leur taux et localisation subcellulaire sont finement régulés par des kinases et phosphatases, dont MTM1 [3].

Dans la fibre musculaire striée squelettique, MTM1 apparaît localisée au niveau d'une zone d'interface entre la membrane plasmique et la membrane du réticulum sarcoplasmique (RS) appelée la triade [4], qui est le lieu du couplage excitation-contraction (CEC). Le CEC permet la libération de $\mathrm{Ca}^{2+} \mathrm{du} \mathrm{RS}$ par des canaux calciques qui sont les récepteurs à la ryanodine (RyRs), en réponse à la dépolarisation (potentiel d'action) de la membrane plasmique induite par l'influx nerveux. L'efflux massif de $\mathrm{Ca}^{2+}$ du RS vers le cytosol produit une augmentation de $\mathrm{Ca}^{2+}$ cytosolique (transitoire calcique) qui déclenche l'activation des protéines contractiles [5]. Le bon fonctionnement du CEC est indispensable à la fonction musculaire et toute altération de ce processus 
peut évidemment avoir des conséquences dramatiques et compromettre la survie de l'organisme.

En 2009, une étude avait révélé l'importance cruciale de MTM1 dans la fonction du CEC du muscle squelettique [6] : en effet, les fibres musculaires de souris Mtm1-KO présentent un défaut du CEC caractérisé par une réduction sévère de l'amplitude des transitoires calciques, associée à une désorganisation des tubules-t (Figure 1B). Cette altération du $\mathrm{CEC}$ est probablement une cause majeure de la faiblesse musculaire fatale chez les patients atteints de la myopathie myotubulaire. Cependant, malgré de nombreux travaux, la fonction primaire de la myotubularine et surtout les mécanismes pathologiques à l'origine de la myopathie restaient controversés.

Le présent projet, récompensé par le prix Master 2015 de la Société Française de Myologie, a visé à caractériser les altérations du CEC dans les fibres musculaires de souris Mtm1-KO, et à tester le rôle d'une altération du métabolisme des PtdInsPs comme mécanisme pathologique. Dans ce travail nous avons utilisé une combinaison de microscopie confocale et d'électrophysiologie afin d'enregistrer les transitoires calciques en réponse à des dépolarisations de la membrane plasmique dans des fibres musculaires Mtm1-KO et contrôles (WT). Nous montrons que les fibres musculaires de souris Mtm1-KO présentent une réduction de $60 \%$ de l'amplitude maximale ainsi qu'un retard de 10 à 30 ms de l'efflux de $\mathrm{Ca}^{2+}$ par le RS (Figure 1C) [7]. Ainsi le flux calcique dans les fibres malades présente non seulement un défaut d'amplitude mais aussi de cinétiques d'activation. Par ailleurs, l'analyse des images confocales des transitoires calciques a révélé que ces altérations sont spatialement hétérogènes dans les fibres musculaires malades, avec certaines régions présentant des déficits exacerbés du transitoire calcique [7]. Nous avons trouvé que ces altérations du flux calcique n'étaient pas systématiquement corrélées à des altérations structurales détectables du réseau de tubules-t, suggérant qu'un mécanisme pathologique autre que des défauts de structure, opère dans les fibres Mtm1-KO. Étant donné que MTM1 possède une activité PtdIns phosphatase, nous avons fait l'hypothèse suivant laquelle, en son absence, l'accumulation de ses substrats serait délétère pour la fonction du CEC. Pour le vérifier, nous avons testé l'effet de l'inhibition in vitro de l'activité PtdIns 3-kinase sur les transitoires calciques. L'exposition des fibres Mtm1-KO à des pan-inhibiteurs pharmacologiques améliore les anomalies du signal calcique, alors qu'elle est sans effet
A

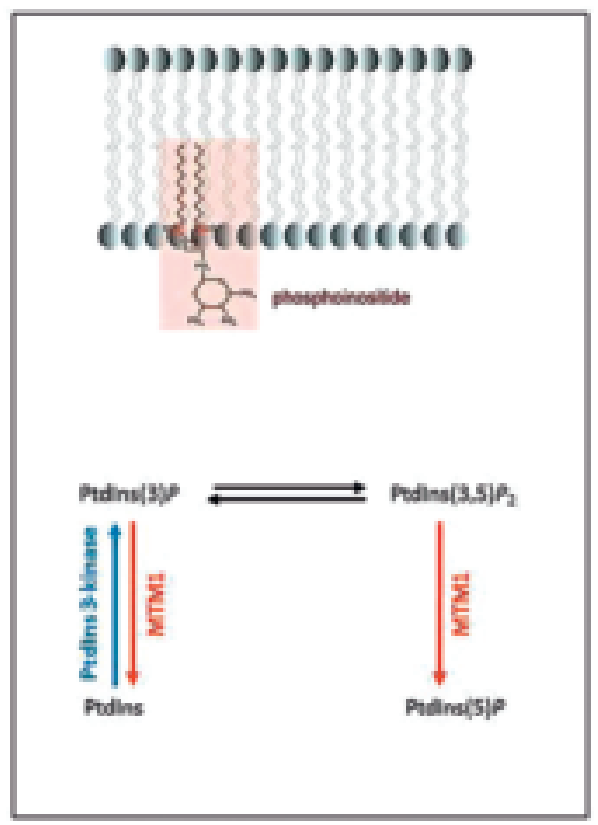

B
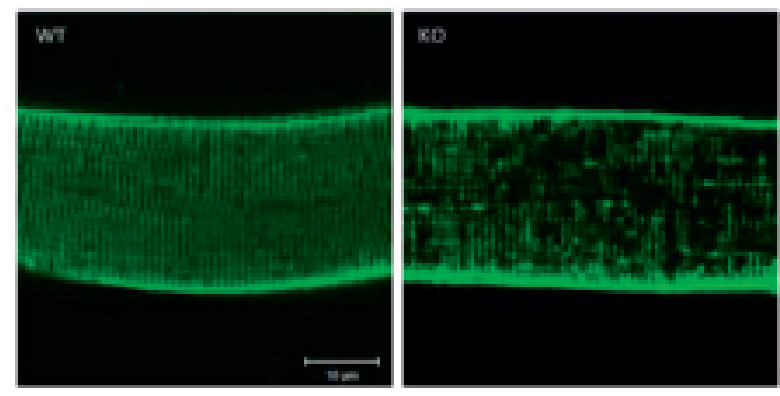

C

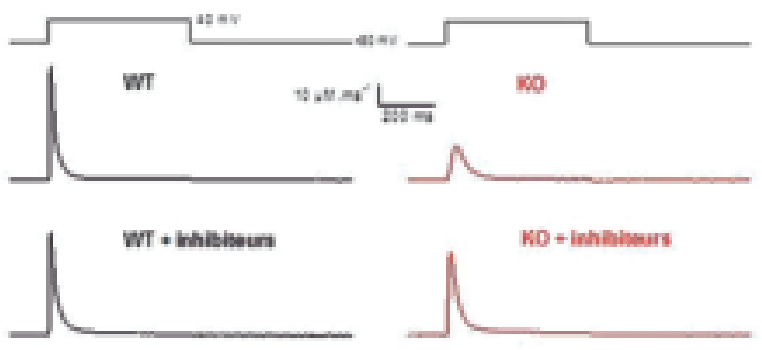

Figure 1

A. Représentation d'un phosphoinositide inséré dans une membrane via ses deux chaînes d'acides gras. MTM1 déphosphoryle le PtdIns(3)P et le PtdIns(3,5)P2 en position 3 du cycle inositol. À l'inverse, la PtdIns 3-kinase produit le PtdIns(3)P à partir du PtdIns.

$\mathrm{B}$. Images du réseau de tubules transverses dans une fibre WT et Mtm1-KO, visualisé avec un marqueur membranaire fluorescent.

C. Exemples du flux calcique du RS déclenché par une dépolarisation de -80 à $+40 \mathrm{mV}$ dans des fibres contrôles (WT) et Mtm1-KO, avec ou sans traitement pharmacologique aux pan-inhibiteurs d'activité PtdIns 3-kinase. 
sur les fibres WT (Figure 1C) [7]. Un traitement prolongé n'a pas amélioré davantage ces paramètres, signifiant que l'effet bénéfique est rapide, et n'implique pas de remodelage structural. Par ailleurs, l'exposition de fibres Mtm1-KO à des inhibiteurs spécifiques de l'activité PtdIns 3-kinase de classe III n'a eu aucun effet sur le signal calcique [7]. Dans l'ensemble, nos résultats indiquent que les défauts du CEC dans les fibres Mtm1-KO ne sont pas uniquement liés à des altérations de structure, mais aussi à un déséquilibre d'activité PtdIns 3-kinase de classe I et/ou de classe II.

Enfin, les résultats bénéfiques obtenus sur la signalisation calcique des fibres musculaires Mtm1-KO nous ont encouragé à tester l'effet de l'inhibition de l'activité Ptdlns 3-kinase in vivo. Le traitement avec un pan-inhibiteur de PtdIns 3-kinase augmente significativement l'espérance de vie des souris Mtm1-KO [7], avec, par exemple, comme montré sur la figure 2, une souris toujours en vie à l'âge de 6 mois. Dans l'ensemble, ces résultats suggèrent que les défauts du CEC dans les fibres musculaires Mtm1-KO sont, au moins en partie, liés à un déséquilibre du métabolisme des PtdInsPs et fournissent une preuve de concept d'approche thérapeutique basée sur l'inhibition de l'activité PtdIns 3-kinase.

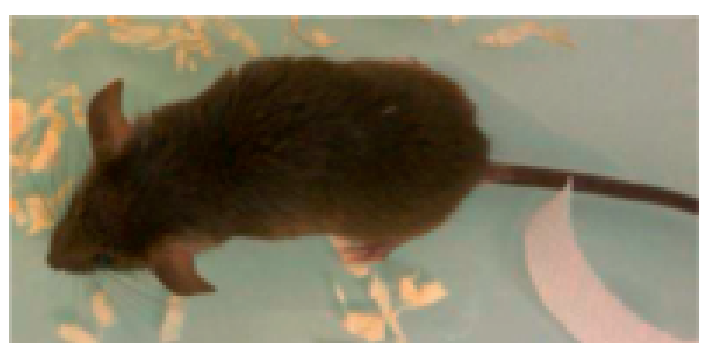

Figure 2

Souris Mtm1-KO traitée avec un pan-inhibiteur d'activité Ptdlns 3-kinase, âgée de 6 mois.

PtdIns 3-kinase inhibition: a potential pharmacological treatment for myotubular myopathy

\begin{abstract}
Myotubular myopathy is a fatal pediatric disease due to mutations in the gene encoding the phosphoinositide 3-phosphatase MTM1.The disease is characterized by severe muscle weakness at birth and respiratory distress, leading to death of most patients within the first year of life. The
\end{abstract}

MTM1-deficient (Mtm1-KO) mouse model reproduces the main features of the human disease including muscle weakness leading to the death after 8 weeks of life. In a recent work, we identified functional alterations of Mtm1-KO muscle fibers that play a critical role in the pathology. Moreover, we showed the beneficial effect of a pharmacological treatment. More precisely, we revealed that disease-associated calcium signaling alterations are heterogeneous at the subcellular level, and that they affect not only the amplitude but also the kinetics of activation of the $\mathrm{Ca}^{2+}$ release flux that triggers contraction. Moreover, we demonstrated that pharmacological inhibition of phosphoinositide 3-kinase activity substantially rescues $\mathrm{Ca}^{2+}$ signaling defects in vitro and prolongs life expectancy of MTM1 deficient mice. Altogether, our results have revealed that altered phosphoinositide metabolism plays a critical role in the pathology and they provide a proof of concept for the use of phosphoinositide 3-kinase inhibitors in myotubular myopathy treatment.

Key words: myopathie myotubulaire - phosphoinositides - couplage excitation-contraction - signalisation calcique - PtdIns 3-kinase

\section{LIENS D'INTÉRÊT}

Les auteurs déclarent n'avoir aucun lien d'intérêt concernant les données publiées dans cet article.

\section{RÉFÉRENCES}

1. Wallgren-Pettersson C, Clarke A, Samson F, et al. The myotubular myopathies: differential diagnosis of the $\mathrm{X}$ linked recessive, autosomal dominant, and autosomal recessive forms and present state of DNA studies. $J$ Med Genet 1995 ; 32 : 673-9.

2. Buj-Bello A, Laugel V, Messaddeq N, et al. The lipid phosphatase myotubularin is essential for skeletal muscle maintenance but not for myogenesis in mice. Proc Natl Acad Sci USA 2002 ; 99 : 15060-5.

3. Balla T. Phosphoinositides: tiny lipids with giant impact on cell regulation. Physiol Rev 2013; 93 : 1019-37.

4. Buj-Bello A, Fougerousse F, Schwab Y, et al. AAV-mediated intramuscular delivery of myotubularin corrects the myotubular myopathy phenotype in targeted murine muscle and suggests a function in plasma membrane homeostasis. Hum $\mathrm{Mol}$ Genet $2008 ; 17: 2132-43$.

5. Dulhunty A. F. Excitation-contraction coupling from the 1950s into the new millennium. Clin Exp Pharmacol Physiol 2006 ; 33 : 763-72.

6. Al-Qusairi L, Weiss N, Toussaint A, et al. T-tubule disorganization and defective excitation-contraction coupling in muscle fibers lacking myotubularin lipid phosphatase. Proc Natl Acad Sci USA 2009; 106 : 18763-8.

7. Kutchukian C, Lo Scrudato M, Tourneur Y, et al. Phosphatidylinositol 3-kinase inhibition restores $\mathrm{Ca} 2+$ release defects and prolongs survival in myotubularin-deficient mice. Proc Natl Acad Sci USA 2016 ; 113 : 14432-7.

Abréviations

Mtm1 : myotubularine; PtdInsP : phosphoinositide; CEC : couplage excitation-contraction ; RyR : récepteur à la ryanodine; DHPR : récepteur des dihydropyridines 